\title{
Efeito do Número de Inseminações Artificiais sobre a Fertilidade de Éguas Inseminadas com Sêmen Diluído, Resfriado a $14^{\circ} \mathrm{C}$ e Transportado ${ }^{1}$
}

\section{Guilherme Ribeiro Valle ${ }^{2}$, José Monteiro da Silva Filho $^{3}$, Maristela Silveira Palhares ${ }^{3}$, Ivan Barbosa Machado Sampaio ${ }^{3}$, Henrique Nunes de Oliveira ${ }^{4}$, José Eduardo Valle Santos ${ }^{2}$}

\begin{abstract}
RESUMO - Estudou-se o efeito do número de inseminações sobre a fertilidade de éguas inseminadas, três vezes/semana (segundas, quartas e sextas-feiras), com sêmen diluído, resfriado e transportado, de apenas um garanhão. As éguas foram inseminadas a partir da detecção, pela palpação retal, de um folículo de 3,0 a 3,5 cm de diâmetro, em um dos ovários, até a ovulação. Utilizaram-se para o transporte o sêmen diluído no diluidor leite desnatado-glicose e o conteiner "Celle" modificado, sendo a dose inseminante de 400 x $10^{6}$ espermatozóides móveis, no momento da diluição final, pré-resfriamento. De acordo com o número de inseminações artificiais (IA) utilizadas/ciclo, os resultados de 148 ciclos, de 100 éguas, foram agrupados em: 1 IA, 2IA, 3 IA e 4 ou mais IA. A eficiência de prenhez foi de 4,29; 5,04; 5,67; e 3,43, para 1,2, 3 e 4 ou mais IA, respectivamente. As características foliculares diferiram em relação à freqüência de inseminações com os maiores valores observados no grupo de éguas inseminadas quatro ou mais vezes. Concluiu-se que menor velocidade de crescimento folicular e maior diâmetro do folículo ovulatório estiveram associados a maior número de inseminações artificiais/ciclo. Na presença de uma concentração espermática adequada, o número de inseminações/ciclo não exerceu influência sobre a fertilidade.
\end{abstract}

Palavras-chave: eqüino, inseminação artificial, número de inseminações, fertilidade

\section{Effect of Artificial Insemination Number on the Fertility in Mares Inseminated with Diluted, Cooled at $14^{\circ} \mathrm{C}$ and Transported Semen}

\begin{abstract}
The effect of the number of insemination on the fertility of mares inseminated three times/week (Monday, Wednesday and Friday), with extended, cooled and transported semen, from only one stallion, was studied. The mares were inseminated from the detection, by rectal palpation, of a 3.0 to $3.5 \mathrm{~cm}$ follicle diameter, in one of the ovaries, until ovulation. The semen was transported in skim milk-glucose extender in a "Celle" modified container; with inseminate doses of $400 \times 10^{6}$ motile spermatozoa at the moment of the final dilution, before cooling. The results of 148 cycles of 100 mares were grouped, in accordance with the number of artificial inseminations (AI), in: 1AI, 2AI, 3AI and 4 or more AI. The pregnancy efficiency was $4.29 ; 5.04 ; 5.67$ and 3.43 to groups $1,2,3$ and 4 or more, respectively. The follicular characteristics were different according to insemination frequency with the greatest values observed on the group of 4 or more times inseminated mares. The results of this experiment permit to conclude that a slow follicular growth speed and the biggest ovulatory follicular diameter were associated with a greater number of artificial insemination /cycle. Moreover, with an appropriate spermatic concentration, the insemination number/cycle have no influence upon fertility.
\end{abstract}

Key Words: equine, artificial insemination, inseminations number, fertility

\section{Introdução}

A freqüência de inseminações ou cobrições é uma variável que tem merecido limitada atenção da maioria dos pesquisadores. Poucos estudos bem delineados, com controles apropriados, têm sido encontrados na literatura. A maioria dos trabalhos tem recomendado inseminações em dias alternados, a partir do segundo ou do terceiro dia do cio, na ausência do controle folicular. Quando submetidas à palpação retal, ou controle folicular pela ultra- sonografia, as éguas têm sido inseminadas a partir da detecção de um folículo de $35 \mathrm{~mm}$ de diâmetro, até a comprovação da ovulação.

A redução do número de IAs/ciclo poderia ser um fator de diminuição de custos num programa de IA de eqüinos, notadamente naqueles envolvendo transporte de sêmen entre haras. Para tal, seriam necessários maiores conhecimentos a respeito do momento da ovulação em relação ao restante do ciclo estral, ao tamanho do folículo à ovulação e à viabilidade espermática no sistema genital feminino.

\footnotetext{
${ }^{1}$ Fonte financiadora: Fundação de Amparo à Pesquisa do Estado de Minas Gerais - FAPEMIG - CAG 188095. Apoio: Regimento de Cavalaria Alferes Tiradentes - PMMG.

2 Aluno de pós-graduação.

3 Professor - Escola de Veterinária - UFMG - Caixa Postal 567 - CEP 30161-970 - Belo Horizonte - MG.

${ }^{4}$ Professor - Departamento de Zootecnia - FMVZ - UNESP - Botucatu - SP.
} 
Ao número de inseminações artificiais, por ciclo estral, tem sido atribuída, algumas vezes, a responsabilidade por piores resultados de fertilidade, o que se justificaria pela maior contaminação uterina veiculada pelo sêmen. No entanto, técnicas adequadas de inseminação, com controle microbiológico, podem eliminar tal preocupação (PICKETT et al., 1987). Por outro lado, repetidas inseminações poderiam aumentar a disponibilidade de espermatozóides, no reservatório espermático, para o momento da ovulação (GINTHER, 1992). Entretanto, os resultados da literatura são conflitantes tanto em relação ao número de IA/ciclo, quanto ao momento dessas inseminações ou montas, em relação ao momento da ovulação.

O objetivo deste trabalho foi verificar os efeitos do número de inseminações artificiais, por ciclo estral, sobre a fertilidade de éguas.

\section{Material e Métodos}

O trabalho foi desenvolvido durante duas estações de monta consecutivas, sendo utilizados 148 ciclos estrais de 100 éguas mestiças de 3 a 19 anos de idade, pertencentes a diferentes categorias reprodutivas (éguas solteiras, éguas paridas e potras). Antes do início de cada uma das estações de monta, todas as éguas solteiras e potras foram submetidas a exame ginecológico, constituído de avaliação conformacional externa e interna do sistema genital, citologia e cultura de conteúdo uterino (exceto as potras) e antibiograma, quando o resultado da respectiva cultura o indicava. A partir dos resultados obtidos, foram realizados tratamentos médico-cirúrgicos indicados.

Os controles de crescimento folicular nas éguas foram realizados por palpação retal em dias alternados, até que se detectasse um folículo de 2,0 a $2,5 \mathrm{~cm}$ de diâmetro em um dos ovários, sendo, a partir daí, realizados diariamente. Ao se detectar um folículo de 3,0 a 3,5cm de diâmetro, iniciaram-se as inseminações artificiais até a ovulação. As éguas paridas foram palpadas a partir do quinto dia após o parto. O diagnóstico de gestação foi feito por palpações retais aos 17 , $21,25,30,35,40,45,60$ e 90 dias após a ovulação.

Foi utilizado, nas duas estações, um mesmo garanhão da raça Brasileira de Hipismo (7-8 anos de idade) como doador de sêmen, que apresentava bom índice de fertilidade, aferida anteriormente (SILVA FILHO et al., 1993).

O sêmen foi colhido por meio de uma vagina artificial modelo Hannover, às segundas, quartas e sextas-feiras. Após avaliação de motilidade e vigor em microscópio óptico e concentração por contagem em câmara de Newbauer, o sêmen foi diluído, a $37^{\circ} \mathrm{C}$, em diluidor à base de leite em pó desnatado-glicose (KENNEY et al, 1975). As doses inseminantes foram constituídas por 400 milhões de espermatozóides móveis em $15 \mathrm{~mL}$ de sêmen diluído e transportadas em container modelo "Celle" modificado (VALLE, 1997), à temperatura final de $14^{\circ} \mathrm{C}$. As éguas encontravam-se em outro município, a $60 \mathrm{~km}$ do município onde se encontrava o garanhão.

De acordo com o número de inseminações recebidas por ciclo, os resultados de 148 ciclos de 100 éguas inseminadas foram agrupados em: 1 IA, 2 IA, 3 IA e 4 ou mais IA.

Com objetivo de obter maior eficiência na detecção de diferenças entre os grupos experimentais, análises de variância foram utilizadas para os resultados de fertilidade obtidos. Por se tratar de uma variável qualitativa, realizou-se a conversão dos dados em quantitativos, segundo proposição de VOSS et al. (1975), obtendo-se um valor numérico médio para cada grupo experimental, chamado de eficiência de prenhez. Tais valores foram obtidos atribuindo-se pontuação a cada ciclo estral (10 pontos para gestação no primeiro ciclo da égua; 8 pontos para gestação no segundo ciclo da égua; 6 pontos para gestação no terceiro ciclo da égua; 4 pontos para gestação no quarto ciclo da égua; e 0 ponto para ciclo não resultando em gestação). A comparação entre as médias foi feita pelo teste de Student-Newman-Keuls (SNK). Valores quantitativos foram também submetidos à análise de variância e teste SNK. Os dados proporcionais (ciclos/concepção, ciclos/égua gestante, taxa de concepção/ciclo e taxa de concepção total) foram submetidos ao teste de qui-quadrado para detecção de diferenças entre os grupos.

\section{Resultados e Discussão}

Os grupos formados de acordo com o número de IA/ ciclo foram semelhantes, quanto às taxas de concepção ao primeiro ciclo e taxas de concepção/ciclo, conforme demonstrado na Tabela 1. Outros parâmetros de eficiência reprodutiva, como número de ciclos/concepção e eficiência de prenhez, também foram semelhantes entre os grupos, conforme Tabela 2.

Parâmetros de controle, como idade, volume de sêmen, volume de diluidor, tempo da colheita ao resfriamento e tempo de resfriamento, foram semelhantes entre os grupos. Entretanto, a temperatura final do sêmen e tempo da colheita à IA foram 
Rev. bras. zootec.

Tabela 1 -Efeito do número de inseminação artificial sobre a fertilidade, por ciclo estral, de éguas inseminadas com sêmen diluído, resfriado e transportado

Table 1 - Effect of the number of artificial insemination on fertility, by estral cycle, of inseminated mares with diluted, cooled and transported semen

\begin{tabular}{|c|c|c|c|c|c|c|c|c|}
\hline \multirow[b]{3}{*}{$\begin{array}{l}\text { Ciclo } \\
\text { Cycles }\end{array}$} & \multicolumn{8}{|c|}{$\begin{array}{l}\text { Grupo } \\
\text { Group }\end{array}$} \\
\hline & \multicolumn{2}{|c|}{$\begin{array}{l}1 \mathrm{IA} \\
1 \mathrm{AI}\end{array}$} & \multicolumn{2}{|c|}{$\begin{array}{l}2 \text { IA } \\
2 \text { AI }\end{array}$} & \multicolumn{2}{|c|}{$\begin{array}{c}3 \mathrm{IA} \\
3 A I\end{array}$} & \multicolumn{2}{|c|}{$\begin{array}{c}4 \text { ou + IA } \\
4 \text { or }+A I\end{array}$} \\
\hline & $\begin{array}{l}\text { Número de } \\
\text { ciclos } 1 \\
\text { Number } \\
\text { ofcycles }\end{array}$ & $\begin{array}{c}\text { Taxade } \\
\text { concepção }^{2} \\
\text { Conception } \\
\text { rate }\end{array}$ & $\begin{array}{l}\text { Número de } \\
\text { ciclos } 1 \\
\text { Number } \\
\text { of cycles }\end{array}$ & $\begin{array}{c}\text { Taxade } \\
\text { concepção }^{2} \\
\text { Conception } \\
\text { rate }\end{array}$ & $\begin{array}{l}\text { Número de } \\
\text { ciclos } 1 \\
\text { Number } \\
\text { of cycles }\end{array}$ & $\begin{array}{c}\text { Taxade } \\
\text { concepção }^{2} \\
\text { Conception } \\
\text { rate }\end{array}$ & $\begin{array}{l}\text { Número } \\
\text { ciclos }{ }^{1} \\
\text { Number } \\
\text { of cycles }\end{array}$ & $\begin{array}{c}\text { Taxade } \\
\text { concepção }^{2} \\
\text { Conception } \\
\text { rate }\end{array}$ \\
\hline 1 & $16(9)$ & 56,25 & $45(26)$ & 57,78 & $26(15)$ & 57,69 & $13(6)$ & 46,15 \\
\hline 2 & $4(0)$ & 0,00 & $19(12)$ & 63,16 & $5(4)$ & 80,00 & $5(0)$ & 0,00 \\
\hline 3 & - & - & $6(2)$ & 33,33 & $1(1)$ & 100,00 & $3(2)$ & 66,67 \\
\hline 4 & $1(0)$ & 0,00 & $1(0)$ & 0,00 & $1(0)$ & 0,00 & - & - \\
\hline 5 & - & - & $2(0)$ & 0,00 & - & - & - & - \\
\hline Total & 21(9) & 42,86 & $73(40)$ & 54,79 & $33(20)$ & 60,61 & $21(8)$ & 38,10 \\
\hline
\end{tabular}

1 Os números entre parênteses referem-se a éguas gestantes.

2 Valores em \%.

IA (inseminações artificiais/ciclo)

1 Number between parenthesis report to pregnancy mares.

2 Values at $\%$.

Al (artificial inseminations/cycle).

diferentes $(\mathrm{p}<0,05)$ entre os grupos (Tabela 2). As diferenças entre os grupos, nesses parâmetros de controle, foram devidas exclusivamente ao acaso, visto que todos os ciclos do experimento foram submetidos às mesmas condições experimentais.

Em decorrência da metodologia utilizada (inseminações às segundas, quartas e sextas-feiras, podendo ocorrer inseminações pós-ovulação até 24 horas após sua detecção), o intervalo das últimas inseminações e a ovulação poderiam ser 24P (24 horas pré-ovulação), 48P (48 horas pré-ovulação), 48PP (48 horas pré-ovulação e outra até 24 horas pós-ovulação), e 72PP (72 horas pré-ovulação e outra até 24 horas pós-ovulação). No trabalho de SILVA FILHO et al. (1998), as taxas de concepção ao primeiro ciclo, concepção/ciclo e eficiência de prenhez do mesmo rebanho não diferiram quanto ao intervalo IA/ovulação e foram, respectivamente: $24 \mathrm{P}(63,3 \% ; 54,8 \%$; e 5,24$)$, 48P (50,0\%; 39,4\%; e 3,64), 48PP (51,3\%; 55,6\%; e $5,11)$ e $72 \mathrm{PP}(60,0 \% ; 55,6 \%$; e 5,11$)$. Portanto, apesar de diferentes intervalos IA/ovulação terem ocorrido entre os grupos de número de IA/ciclo (Tabela 2), estes não constituíram fator de interferência nos resultados obtidos no presente experimento.

Os parâmetros tamanho do folículo à ovulação $\mathrm{e}$ tempo de crescimento do folículo de $2,75 \mathrm{~cm}$ de diâmetro até à ovulação, podem ser considerados como resultados dos tratamentos, uma vez que, quanto maior o número de IAs, maiores o tamanho do folículo e o tempo de crescimento do folículo de $2,75 \mathrm{~cm}$ de diâmetro até à ovulação. Por consequiência, observou-se diferença $(\mathrm{p}<0,05)$ entre os grupos experimentais quanto a esses parâmetros (Tabela 2). Como as IAs iniciavam-se sempre a partir de determinado tamanho folicular (3,0 a 3,5 cm de diâmetro), ciclos com folículos maiores à ovulação tenderam a receber maior número de IAs (Tabela 2). Isso suporta a hipótese de associação entre as duas variáveis, ou seja, folículos maiores à ovulação demorariam mais tempo para crescer que folículos menores.

Ao excessivo número de IAs/ciclo são atribuídos, muitas vezes, piores resultados de fertilidade em eqüinos, a qual decorre da maior veiculação de contaminantes ao útero (PICKETT et al., 1987).

O número de IAs/ciclo foi, segundo a metodologia utilizada neste trabalho, dependente da velocidade de crescimento folicular, já que as IAs foram definidas pelo tamanho folicular. No entanto, a velocidade de crescimento folicular também determina o tempo de duração do cio, estabelecendo uma relação entre o número de IAs/ciclo e a duração do cio.

Assim, PICKETT et al. (1974), procurando reduzir a duração do cio pela aplicação de hCG, obtiveram êxito, resultando melhor fertilidade para o grupo com menor duração de cio, acompanhado por menor número de IAs. Por outro lado, VOSS et al. (1982) observaram que a duração do cio de éguas inseminadas que ficaram gestantes foi maior $(7,9$ dias) que a de éguas que ficaram vazias (6,4 dias). Já LIMA (1995) obteve resultados semelhantes de fertilidade para três diferentes agrupamentos de éguas quanto à duração do cio avaliado pela rufiação, que 
Tabela 2 - Efeito do número de inseminação artificial sobre parâmetros reprodutivos de éguas inseminadas com sêmen diluído, resfriado e transportado

Table 2 - Effect of the number of artificial insemination on reproductive aspects of inseminated mares with diluted, cooled and transported semen Característica avaliada

Evaluated trait

\begin{tabular}{|c|c|c|c|c|}
\hline \multirow{3}{*}{ - } & \\
\hline & $1 \mathrm{IA}$ & 2 IA & 3 IA & $4 \mathrm{ou}+\mathrm{IA}$ \\
\hline & $1 \mathrm{AI}$ & $2 A I$ & $3 A I$ & $4 o r+A I$ \\
\hline Número de ciclos & 21 & 73 & 33 & 21 \\
\hline \multicolumn{5}{|l|}{ Number of cycles } \\
\hline Idade (anos) & $10,33 \pm 3,92$ & $11,27 \pm 4,53$ & $8,94 \pm 4,66$ & $11,86 \pm 4,41$ \\
\hline \multicolumn{5}{|l|}{ Age (years) } \\
\hline $\mathrm{N}^{\circ} \mathrm{IA} /$ ciclo & $1,00^{\mathrm{a}}$ & $2,00^{\mathrm{b}}$ & $3,00^{\mathrm{c}}$ & $5,48 \pm 2,25^{\mathrm{d}}$ \\
\hline \multicolumn{5}{|l|}{ Number of AI/cycle } \\
\hline $\mathrm{N}^{\mathrm{o}}$ ciclos/concepção & 2,33 & 1,83 & 1,65 & 2,63 \\
\hline \multicolumn{5}{|l|}{ Number of cycle/conception } \\
\hline Taxa de concepção/ciclo & 0,43 & 0,55 & 0,61 & 0,38 \\
\hline \multicolumn{5}{|l|}{ Conception/cycle rate } \\
\hline Eficiência de prenhez & 4,29 & 5,04 & 5,67 & 3,43 \\
\hline \multicolumn{5}{|l|}{ Pregnancyefficiency } \\
\hline Intervalo IA/ovulação * & $1,75 \pm 0,44^{\mathrm{a}}$ & $2,21 \pm 1,14^{\mathrm{ab}}$ & $2,67 \pm 0,92^{b}$ & $2,76 \pm 0,77^{b}$ \\
\hline \multicolumn{5}{|l|}{ AI/ovulation interval * } \\
\hline Volume de sêmen (mL) & $3,94 \pm 1,43$ & $3,59 \pm 1,46$ & $3,87 \pm 1,74$ & $4,19 \pm 1,72$ \\
\hline \multicolumn{5}{|l|}{ Semen volume $(m L)$} \\
\hline Volume de diluidor (mL) & $11,06 \pm 1,44$ & $11,47 \pm 1,21$ & $11,14 \pm 1,74$ & $10,81 \pm 1,72$ \\
\hline \multicolumn{5}{|l|}{ Extender volume $(m L)$} \\
\hline Tempo coleta/resfriamento (min) & $53,05 \pm 8,10$ & $47,84 \pm 9,95$ & $47,50 \pm 13,55$ & $48,63 \pm 13,93$ \\
\hline \multicolumn{5}{|l|}{ Collection/cooling time ( $\mathrm{min})$} \\
\hline Tempo de resfriamento (min) & $236,43 \pm 57,98$ & $230,69 \pm 52,90$ & $227,74 \pm 57,71$ & $217,47 \pm 48,51$ \\
\hline \multicolumn{5}{|l|}{ Cooling time (min) } \\
\hline Temperatura do sêmen $\left({ }^{\circ} \mathrm{C}\right)$ & $12,36 \pm 2,17^{b}$ & $14,22 \pm 1,80^{\mathrm{a}}$ & $13,45 \pm 1,90^{\mathrm{a}}$ & $13,79 \pm 1,95^{\mathrm{a}}$ \\
\hline \multicolumn{5}{|l|}{ Semen temperature $\left({ }^{\circ} \mathrm{C}\right)$} \\
\hline Tempo rufiação/IA(min) & $35,77 \pm 18,53$ & $38,93 \pm 18,60$ & $33,05 \pm 15,86$ & $33,30 \pm 15,14$ \\
\hline \multicolumn{5}{|l|}{ Teasing/AI time(min) } \\
\hline Tempo colheita/IA (min) & $319,48 \pm 69,44^{\mathrm{a}}$ & $303,41 \pm 59,53^{a b}$ & $297,70 \pm 63,73^{\mathrm{ab}}$ & $285,15 \pm 58,06^{b}$ \\
\hline \multicolumn{5}{|l|}{ Collection/AItime (min) } \\
\hline Folículo à ovulação (cm) & $3,68 \pm 0,57^{\mathrm{a}}$ & $3,75 \pm 0,48^{\mathrm{ab}}$ & $3,75 \pm 0,41^{\mathrm{ab}}$ & $4,04 \pm 0,61^{b}$ \\
\hline Ovulation follicle size $(\mathrm{cm})$ & & & & \\
\hline $\begin{array}{l}\text { Folículo } 2,75 \text { à ovulação (dias) } \\
2.75 \text { follicle to ovulation (days) }\end{array}$ & $4,08 \pm 2,23^{\mathrm{a}}$ & $4,25 \pm 1,34^{\mathrm{a}}$ & $7,20 \pm 1,82^{b}$ & $12,13 \pm 6,08^{c}$ \\
\hline
\end{tabular}

* $\quad$ Significado apenas para demonstrar similaridade entre os grupos (1 = 24P; última IA 24 h pré-ovulação); (2 = 48P; última IA 48 h préovulação); 3 = 48PP; últimas IAs $48 \mathrm{~h}$ pré $+24 \mathrm{~h}$ pós-ovulação); 4 = 72PP; últimas IAs 72 h pré- + 24 h pós-ovulação).

a, b, c, d Números dentro de linhas, seguidos por letras desiguais, diferem $(P<0,05)$.

IA (inseminação artificial).

* $\quad$ Significance only to demonstrate similarity between groups (1=24P; last $\mathrm{Al} 24 \mathrm{~h}$ pre-ovulation); (2=48P; last $\mathrm{Al}$ 48h pre-ovulation); 3 = 48PP; last Als $48 \mathrm{~h}$ pre +24 h post-ovulation); ( $4=72 P P$; last Als 72 h pre +24 h post-ovulation $)$.

a, $b, c, d \quad$ Numbers within rows, followed by different letters, differ $(P<.05)$

Al (artificialinsemination).

foram de $53,85 \%$ até 5 dias de cio; $52,17 \%$ entre 5 e 7 dias de cio; e $66,67 \%$ acima de 7 dias de cio.

CARVALHO (1994), SILVA FILHO (1994) e LIMA (1995), em seus respectivos experimentos, não obtiveram diferenças entre número de IA/ciclogestante e número de IA/ciclo vazio. Além disso, LIMA (1995) também verificou que o número de IA não teve efeito sobre a fertilidade de éguas inseminadas com sêmen resfriado e transportado a $20^{\circ} \mathrm{C}$, de maneira semelhante aos resultados obtidos neste trabalho (Tabelas 1 e 2).

Diferentemente de LIMA (1995), que categorizou seus resultados em uma, duas e três ou mais IAs, no presente experimento, os ciclos foram categorizados em uma, duas, três e quatro ou mais IAs, o que tornou mais precisa a avaliação. Dentro desse contexto, observou-se que $85,81 \%$ dos ciclos receberam de uma a três IAs, sendo uma $(14,19 \%)$, duas $(49,32 \%)$ e três $(22,30 \%$ dos ciclos), enquanto ciclos de quatro IAs contribuíram com 6,76 dos $14,19 \%$ representados pelo grupo de quatro ou mais IAs. Isso mostra que o grupo de três IAs foi bastante expressivo, merecendo ser individualizado como feito nesta pesquisa.

Os resultados dos pesquisadores acima, como no presente experimento, mostram ausência de influên- 
cia do número de IA/ciclo sobre a fertilidade. Já resultados como os de VOSS et al. (1982), PICKETT et al. (1987) e FERREIRA (1993) mostram melhora da fertilidade, com o aumento do número de IAs/ ciclo. Na mesma publicação de PICKETT et al. (1987), outra observação mostrou haver queda da fertilidade, com aumento do número de IA.

Admitindo-se a existência do reservatório espermático na égua, poder-se-ia esperar que, com baixas concentrações de espermatozóides por dose inseminante, sucessivas IAs poderiamestar aumentando, aditivamente, o número de espermatozóides nesse reservatório e, assim, a possibilidade de sucesso na fecundação do ovócito. No entanto, essa possibilidade seria viável mediante a utilização de sêmen de elevada sobrevivência na genitália da égua, pois, se isso não ocorresse, a dose inseminante estaria apenas substituindo os espermatozóides da dose anterior, e não somando-se a eles. Por este raciocínio, esperar-seiam os piores resultados com uma única IA/ciclo. Tal expectativa não se confirmou, possivelmente devido à utilização de adequada concentração espermática. Assim, existe a possibilidade de que o número de IAs/ciclo possa ter efeito sobre a fertilidade, na presença de pequena concentração/dose inseminante, contendo espermatozóides de elevada longevidade no sistema genital feminino, em que haveria efeito aditivo dos espermatozóides da primeira e segunda doses inseminantes.

Nos dois experimentos de VOSS et al. (1982), em que foram feitos grande número de observações (257 éguas no primeiro e 64 no segundo), obteve-se efeito positivo do maior número de IAs sobre a fertilidade. No primeiro experimento, os autores não tiveram controle sobre as doses inseminantes. No entanto, no segundo, foi utilizado sêmen a fresco com doses inseminantes de 100 milhões de espermatozóides, com motilidade progressiva, e IAs a cada 48 horas. Nesse caso, apesar de não se ter informação da qualidade seminal do único garanhão utilizado, a concentração espermática estava no limite mínimo para o sêmen a fresco (PICKETT et al., 1987), e a possibilidade levantada acima pode ter ocorrido.

PICKETT et al. (1987) também obtiveram melhora da fertilidade com o aumento do número de IAs/ ciclo, utilizando dados coletados durante seis anos, sem que, no entanto, tivesse controle da concentração espermática utilizada nas IAs com sêmen a fresco. FERREIRA (1993) observou também melhora da fertilidade com maior número de IAs/ciclo de éguas, inseminadas com sêmen resfriado de jumento, utilizando-se doses inseminantes de 250 milhões de espermatozóides móveis. Foram comparadas uma ou duas IAs/ciclo, e o somatório da segunda à primeira dose inseminante pode ter ocorrido.

A maior veiculação de contaminantes ao útero, em decorrência do maior número de IAs, segundo PICKETT et al. (1987), referindo-se à literatura, não é o caso em condições de assepsia utilizadas na IA, higiene genital do garanhão doador de sêmen e utilização de antibióticos no diluidor. Entretanto, a possibilidade de contaminação uterina, mediante sucessivas intervenções (CASLIK, 1937), é uma realidade e a possibilidade de infecção, considerável. No entanto, intervenções uterinas como as da IA, sob as condições de assepsia deste experimento, não tiveram efeito deletério sobre a fertilidade.

No momento, as possibilidades de se reduzir o número de IAs são, por intermédio da indução da ovulação, por meio de aplicações exógenas de medicamentos que contenham LH ou GnRH (PALMER, 1993), pelo controle folicular tão freqüente como de 12 em 12 horas, com IAs pós-ovulação, e pela utilização de uma estação de monta curta, nos meses de maior luminosidade, acompanhados por estros com períodos mais reduzidos (TRUM, 1950; GINTHER, 1974; e PALHARES, 1989).

\section{Conclusões}

Menor velocidade de crescimento foliculare maior diâmetro do folículo ovulatório estiveram associados a maior número de inseminações artificiais/ciclo. Além disso, na presença de uma concentração espermática adequada, o número de inseminações/ ciclo não exerce influência sobre a fertilidade.

\section{Referências Bibliográficas}

CARVALHO, G.R. Fertilidade do sêmen eqüino diluído, resfriado a $20^{\circ} \mathrm{C}$ e transportado. Viçosa, MG: UFV, 1994 58p. Dissertação (Mestrado em Zootecnia) - Faculdade de Zootecnia/Universidade Federal de Viçosa, 1994.

CASLICK, E.A. 1937. The vulva and the vulvo-vaginal orifice and its relation to the genital health of the thoroughbred mare. Cornell Vet., 27(2):178-187.

FERREIRA, M.F.L. Efeito de diluentes e taxa de resfriamento sobre a motilidade espermática e fertilidade do sêmen de jumento (Equus asinus). Belo Horizonte, MG: UFMG, 1993. 67p. Dissertação (Mestrado em Medicina Veterinária) - Escola de Veterinária/Universidade Federal de Minas Gerais, 1993.

GINTHER, O.J. 1974. Ocurence of anestrus, estrus, diestrus, and ovulation over a 12-month period in mares. Am. J. Vet. Res., 35(9):1173-1179.

GINTHER, O.J. 1992. Reproduction biology of mare (basic and 
apllied aspects). 2.ed., Cross Plains: Equiservices. 642p.

KENNEY, R.M., BERGMAN, R.V., COOPER, W.L. et al. 1975. Minimal contamination techniques breeding mares: technique and preliminary findings. Proceedings of the American Association of Equine Practitioners, 21:327-336.

LIMA, M.C.C. Sêmen eqüino: efeito da diluição, resfriamento à $20^{\circ} \mathrm{C}$ e transporte sobre a fertilidade). Belo Horizonte, MG: UFMG, 1995. 121p. Dissertação (Mestrado em Medicina Veterinária) - Escola de Veterinária/Universidade Federal de Minas Gerais, 1995.

PALHARES, M.S. Avaliação da atividade ovariana e eficiência reprodutiva de potras e éguas da raça Mangalarga Marchador. Belo Horizonte, MG: UFMG, 1989. 89p. Dissertação (Mestrado em Medicina Veterinária) - Escola de Veterinária/Universidade Federal de Minas Gerais, 1989.

PALMER, E. 1993. Induction of ovulation. In: MCKINNON, A.O., VOSS, J.L. (Eds.) Equine reproduction. Malvern: Lea \& Febiger. p.344-347.

PICKETT, B.W., BACK, D.G., BURWASH, L.D. et al. The effect of extenders, spermatozoal numbers and rectal palpation on equine fertility. In: TECHNICAL CONFERENCE ON ARTIFICIAL INSEMINATION AND REPRODUCTION, 5, 1974, Chicago. Proceedings ... Chicago: National Association of Animal Breeders, 1974. p.47-58.

PICKETT, B.W., SQUIRES, E.L., MCKINNON, A.O. 1987. Procedures for collection, evaluation and utilization of stallion semen for artificial insemination. Fort Colins: Colorado State University, Animal Reproduction Laboratory. 125p.

SILVA FILHO, J.M. Aspectos do manejo reprodutivo e do sêmen na inseminação artificial de éguas. Viçosa, MG: UFV, 1994. 497p. Tese (Doutorado em Zootecnia) - Universidade Federal de Viçosa, 1994.
SILVA FILHO, J.M., SATURNINO, H. M., PALHARES, M. S et al. Efeito do intervalo entre as duas últimas inseminações e da concentração espermática sobre a fertilidade de éguas inseminadas com sêmen fresco, diluído. In: CONGRESSO BRASILEIRO DE REPRODUÇÃO ANIMAL, 10, 1993, Belo Horizonte. Anais ... Belo Horizonte: CBRA, p.264 1993.

SILVA FILHO, J.M., VALLE, G.R., SATURNINO, H.M. et al. 1998. Influência do intervalo entre a inseminação artificial com sêmen diluído resfriado e transportado e a ovulação sobre a fertilidade de éguas. Arq. Bras. Med. Vet. Zootec., 50(5): 563-568.

TRUM, B.F. 1950. The estrous cycle of the mare. Cornell Vet., 40(1):17-23.

VALLE, G.R. Efeito da rufiação e manipulação do sistema genital e transporte de sêmen, em container "Celle"modificado, na avaliação de uma técnica de inseminação artificial em eqüinos. Belo Horizonte, MG: UFMG, 1997. 349p. Dissertação (Mestrado em Medicina Veterinária) - Escola de Veterinária/Universidade Federal de Minas Gerais, 1997.

VOSS, J.L., PICKETT, B.W., BACK, D.G. et al. 1975. Effect of rectal palpation on pregnancy rate of nonlactating, normaly cycling mares. J. Anim. Sci., 41(3):829-834.

VOSS, J.L., SQUIRES, E.L., PICKETT, B.W. et al. 1982. Effect of number and frequency of inseminations on fertility of mares. J. Reprod. Fertil., 32:53-57. (Suppl.)

Recebido em: 25/02/99

Aceito em: 20/06/00 